\title{
Novice and Advanced Beginner Nurses' Patient Safety Management Activities: Mediating Effects of Informal Learning
}

\author{
Kim, Nam $\mathrm{Yi}^{\circledR}$ \\ Assistant Professor, Department of Nursing, Daejeon linstitute of Science and Technology
}

Purpose: The purpose of this study is to identify the effects of the organizational factors and nursing competency of novice and advanced beginner nurses on patient safety management activities, and to confirm the mediating effect of informal learning. Methods: Responses to questionnaires from 169 novice and advanced beginner nurses in South Korea were analyzed. For model fit and hypothesis, maximum likelihood method and covariance structure modeling were used, and bootstrapping was used for significance level. Organizational factors and nursing competencies were independent variables. Informal learning and patient safety management activities were mediator and dependent variables, respectively. Results: Informal learning exhibited a partial mediating effect on the relationship between nursing competency and patient safety management activities and completely mediated the relationship between organizational factors and patient safety management activities. Conclusion: In order to improve the patient safety management activities of novice and advanced nurses, there is a need for interventions that can improve organizational changes and individual nursing competency. Based on this, it will be possible to increase patient safety management activities by developing educational programs including informal learning and improving patient safety culture.

Key Words: Learning; Nursing, practical; Patient safety; Safety management

\section{INTRODUCTION}

When providing healthcare services, patient safety is the most important principle for all members of any hospital. Failure to provide patient safety threatens patients' lives, reduces patients' trust in physicians, and prolongs patients' hospital stay [1]. Patient safety is a crucial concern in healthcare because failure to provide such safety not only decreases healthcare quality but also causes financial losses [2]. To enhance patient safety management, hospitals attempt to establish new healthcare cultures and patient safety systems [3]. However, the rate of patient safety accidents continues to increase [4].

Patient safety management activities refers to measures and activities performed to prevent injuries and accidents among patients or systematic activities conducted to reduce, eliminate, and prevent actual and potential risks to patients in healthcare institutions [5]. Approaches to increase patient safety management activities are generally classified as those stressing organizational and human factors, and these two approaches mutually influence each other in complex interactions within hospital environments [6]. Organizational factors can be considered as macroscopic factors, including environments such as organizational structures, policies, cultures, and communication structures [7]. Effective organizational communication positively affects attitudes toward patient safety [8], whereas task overburden and unsuitable human resource management cause job dissatisfaction and interference with effective communication and decision-making, thereby negatively influencing patient safety [9]. To engage in patient safety management activities, in-depth awareness of the patient safety culture is crucial, which is preceded by organizational involvement [10]. The aforementioned discussion indicates that organizational factors are associated with communication, decision-making, attitudes toward patient safety, and awareness of the patient safety culture; furthermore, these factors are crucial in patient

\footnotetext{
Corresponding author: Kim, Nam Yi

Department of Nursing, Daejeon Institute of Science and Technology, 100 Hyecheon-ro, Seo-gu, Daejeon 35408, Korea.

Tel: +82-42-580-6279, Fax: +82-42-586-6493, E-mail: namyi00@naver.com

Received: Jun 3, 2020 | Revised: Aug 5, 2020 | Accepted: Sep 21, 2020

This is an open access article distributed under the terms of the Creative Commons Attribution Non-Commercial License (http://creativecommons.org/licenses/ by-nc/3.0), which permits unrestricted non-commercial use, distribution, and reproduction in any medium, provided the original work is properly cited.
} 
safety management activities.

Personal factors can be regarded as microscopic factors related to an individual's knowledge, skills, attitudes, and perceptions [11]. Nurses are crucial to patient safety management activities and can ensure patient safety through monitoring, surveillance, and management activities [12] However, nursing quality differs depending on nurses' clinical experience, skills and competence, education, and professionalism [13]. In the clinical career stage, considering Korean nurses' level of practice, novice nurses (with less than one year of clinical experience) are immersed in their own work; it is difficult to understand specific situations, and thus they are a necessary step to support learning opportunities or preceptors [14]. Advanced beginner nurses (with 1 3 years of clinical experience) are can easily perform nursing task but are not yet fully capable of making independent judgments and decisions [14] Therefore, if the nursing competency of novice and advanced beginner nurses who cannot perform nursing tasks independently or flexibly are improved, patient safety management activities may also improve [15]. Nursing competency is the combination of knowledge, skill, motivation, and attitude required in clinical settings and refers to intrinsic characteristics that are essential for nurses to manage new environments and perform professional nursing tasks [13]. Despite considerable technological and knowledge advancements, healthcare systems must rely on human intervention. In particular, nurses must perform numerous patient safety management activities, including patient identification, fall prevention, infection management, drug administration, and medical device management. Therefore, enhancing the competence of nursing staff at the novice and advanced beginner levels can impact patient safety management through improved nursing activities.

As novice and advanced beginner nurses do not have sufficient systematic learning and experience compared to proficient nurses [16], their organizational involvement and task performance are influenced by informal learning in clinical settings $[17,18]$. In contrast to official and formal learning of the tasks required for clinical practice, informal learning refers to learning processes that involve performing the related tasks, which facilitates an understanding of the organizational context and formation of interrelationships among organizational members $[19,20]$. Such learning is influenced by the competence of individuals as well as the organization $[19,21]$; moreover, it affects the organizational involvement and task performance of individuals $[17,18]$. Therefore, the present study was done to examine the effect of informal learning on patient safety management activities, which comprise a large component of the nursing job [12]. When a patient safety incident occurs, various improvements must be implemented to prevent the same incident from recurring. Some previous studies in this area have focused on organizations $[9,10,22]$, and others on persons $[13,15]$. The Systems Engineering Initiative for Patient Safety model considers the complexities of interactions within a system [23]. According to this model, humans, tasks, instruments, skills, environment, and organizational circumstances interact with and influence one another, thereby affecting job quality and outcomes [23]. However, limited experimental data are available to identify factors that affect patient safety management activities involving novice and advanced beginner nurses, to analyze the effect of enhanced informal learning, and to understand its impact on patient safety management activities. The purpose of this study was to examine the organizational factors (e.g., task, instrument, and environment), nursing competencies (e.g., human and skill), and the mediating effects of informal learning in relation to patient safety management activities of novice and advanced beginner nurses.

\section{METHODS}

\section{Study Design}

This study was a survey designed to describe and predict patient safety management activities performed by novice and advanced beginner nurses. In this study, organizational factors and nursing competencies were independent variables and informal learning and patient safety management activities were mediator and dependent variables, respectively. The conceptual framework of the study is shown in Figure 1.

\section{Participants}

Three university hospitals in Daejeon, Korea with over 800 beds were conveniently sampled. With the cooperation of the institution, recruitment announcements were made and volunteers were allowed to participate. The self-report questionnaire was conducted for novice and advanced beginner nurses with less than three years of clinical experience [16]. For structural equation modeling, the recommended sample size was $n=10 \sim 20$ participants per observed variable, with a minimum total sample size of $n=150$ if the normality assumption is satisfied [24]. Considering the dropout rate, surveys were distributed to 200 nurses, and 176 surveys were returned (a response rate of 


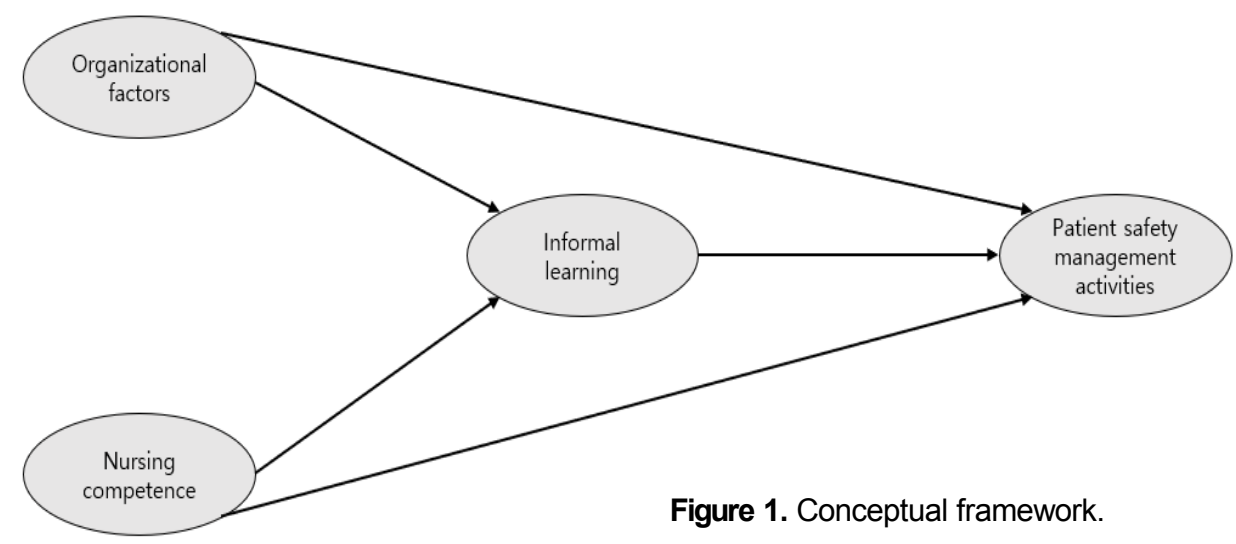

$88 \%)$. After reviewing the returned surveys, seven were excluded because $10 \%$ or more of the data were missing. The final analysis included 169 surveys.

\section{Measurements}

\section{1) Organizational factors}

The organizational factor scale of Kim [7], consisting of safety management, manager values, system and regulation, safety investment, and safety training was used. This scale was comprised of 20 five-point Likert scale items. The higher the score, the higher the organization's level of safety management. In Kim's study, the Cronbach's $\alpha$ 's for safety management, manager values, system and regulation, safety investment, and safety training were $.91, .85, .91, .87$, and .83 [7], in this study, were $.92, .90$, $.90, .83$ and .86 . The overall reliability Cronbach's $\alpha$ for organizational factors was .96 .

\section{2) Nursing competency}

To assess nursing competency, the Nursing Competence Measurement scale developed by Park and Kim [16] was used, and the items were classified into the following factors: knowledge and skill, professionalism, management and leadership, and relationship and cooperation [16]. The scale comprised 30 four-point Likert scale items. The higher the score, the higher the individual's nursing competency. Cronbach's $\alpha$ in Park and Kim's study [16] and in the present study were .92 and .96 , respectively.

\section{3) Informal Learning}

Using the informal learning measurement scale of Moon and Kim [19], the job knowledge acquisition, adjustment to organization and contextual understanding, relationship formation, and personal development skill cultivation were confirmed. The instrument was comprised of 19 five-point Likert scale items. The higher the score, the more informal learning has been completed. The Cronbach's $\alpha$ in Moon and Na's research [19] and the present study were .96 and .89 , respectively.

\section{4) Patient safety management activities}

Park's [25] patient safety management scale was used to measure correct patient identification, effective communication, high-risk medication management, accurate surgery/procedure identification, infection prevention, and fall prevention. This instrument was consistent with the six international patient safety goals and comprised 24 five-point Likert scale items. The higher the score, the higher the level of the patient safety management activity. The Cronbach's $\alpha$ in Park's study [25] and the present study were .89 and .96 , respectively.

\section{Data Collection}

The data were collected from June 1 to July 30, 2019 by administering the structured self-report questionnaire. For survey distribution, the researcher submitted the study proposal and Institutional Review Board (IRB) approval letter to nursing departments at the hospitals and provided information on the purposes and procedure of the study to seek cooperation in recruiting study participants. The participants-nurses with clinical experience of less than 3 years-were informed of the purposes and procedures of the study, voluntary participation, right to withdraw from study, and confidentiality. Those who consented to participate signed a written consent form. Among the 200 distributed surveys, 169 were included in the final analysis.

\section{Data Analysis}

The data were analyzed using SPSS 25.0 (SPSS; IBM, Armonk, NY, USA) and AMOS 21.0 (SPSS Amos; IBM, 
Chicago, IL, USA). The general characteristics of the participants were examined by computing descriptive statistics, whereas normality in distribution was evaluated by examining kurtosis and skewness. The reliability of the instruments was evaluated using Cronbach's $\alpha$, and correlations among measurement variables were examined using Pearson's correlation coefficients. The fitness of the hypothetical model was tested using normed $x^{2}$ values, normed fit index (NFI), non-normed fit index (Tucker-Lewis index, TLI), comparative fit index (CFI), and standardized root mean squared residual (SRMR). The model fitness and hypotheses were evaluated using the maximum likelihood method and covariance structure modeling. Bootstrapping was used to examine the statistical significance of direct, indirect, and total effects in the hypothetical model. The significance level was set at .05 .

\section{Ethical Consideration}

This study was approved by the Gimcheon University Institutional Review Board (IRB GU-201904-HRa-03-02). Participants were informed of the purpose, method, voluntary participation, autonomy of withdrawal, and confidentiality of information. It was conducted after receiving written consent from the nurses who agreed to participate in the study. A consent form and questionnaire were placed in the nurse's lounge for voluntary participation. The completed questionnaire was sealed and retrieved in a collection box. The research was conducted in accordance with the provisions of the Declaration of Helsinki.

\section{RESULTS}

\section{General Characteristics of Participants}

The participants' mean age was $24.35 \pm 3.07$ years. Regarding the clinical career stage, $44.4 \%$ and $55.6 \%$ of the participants were novice nurses $(n=75)$ and advanced beginner nurses $(n=94)$, respectively The average number of safety training session was $2.20 \pm 2.05$ years. The average safety accident experience was $1.36 \pm 0.48$ years (Table 1 ).

\section{Descriptive Statistics and Correlational Analysis with Variables}

The mean values of the measurement variables were $3.84 \pm 0.48$ (of 5 points), $3.08 \pm 0.32$ (of 4 points), $3.80 \pm .048$ (of 5 points), and $4.15 \pm 0.48$ (of 5 points) for the organizational factors, nursing competency, informal learning, and patient safety management activities scales, respectively
Table 1. General Characteristics and Descriptive Statistics of Observed Variables

$(N=169)$

\begin{tabular}{|c|c|c|}
\hline Variables & Categories & $\begin{array}{l}\mathrm{n}(\%) \text { or } \\
\mathrm{M} \pm \mathrm{SD}\end{array}$ \\
\hline Age (year) & & $24.35 \pm 3.07$ \\
\hline Clinical career stage & $\begin{array}{l}\text { Novice } \\
\text { Advanced beginner }\end{array}$ & $\begin{array}{l}75(44.4) \\
94(55.6)\end{array}$ \\
\hline Work department & $\begin{array}{l}\text { Medical } \\
\text { Surgical } \\
\text { Special department }\end{array}$ & $\begin{array}{l}35(20.7) \\
51(30.2) \\
83(49.1)\end{array}$ \\
\hline $\begin{array}{l}\text { Safety education } \\
\text { (number of times) }\end{array}$ & $\begin{array}{l}\text { Yes } \\
\text { No }\end{array}$ & $\begin{array}{r}150(88.8) \\
19(11.2) \\
2.20 \pm 2.05\end{array}$ \\
\hline $\begin{array}{l}\text { Safety accident experience } \\
\text { (number of incidents) }\end{array}$ & $\begin{array}{l}\text { Yes } \\
\text { No }\end{array}$ & $\begin{array}{r}108(63.9) \\
61(36.1) \\
1.36 \pm 0.48\end{array}$ \\
\hline Organizational factors & & $3.84 \pm 0.48$ \\
\hline Nursing competence & & $3.08 \pm 0.32$ \\
\hline Informal learning & & $3.80 \pm 0.48$ \\
\hline $\begin{array}{l}\text { Patient safety } \\
\text { management activities }\end{array}$ & & $4.15 \pm 0.48$ \\
\hline
\end{tabular}

(Table 1).

To test the normality of the sample, the absolute values of skewness and kurtosis were examined. The skewness and kurtosis values ranged from 0.07 to 1.11 and 0.10 to 1.37 , respectively. As the skewness values were lower than 2 , and kurtosis values less than 7 , the normal distribution of the measurement variables was validated (Table 2). The correlations between all pairs of the measurement variables were significant. The absolute values of the correlation coefficients ranged from .50 to .63 , thereby validating that multicollinearity was not a concern. The average variance extracted (AVE) was 0.65 to 0.76 and the construct reliability (CR) was 0.92 to 0.93 , thereby confirming the concentrated validity. The AVE value has increased significantly over $\mathrm{r}^{2}$ (Table 2).

\section{Testing the Fitness of the Hypothetical Model and its Effects}

The index values for testing model fitness were as follows: $x^{2} / \mathrm{DF}=2.17$, AGFI=.84, NFI=.89, TLI=.93, CFI=.94, and SRMR $=.04$. Although the AGFI, NFI did not reach the commonly accepted criterion, the model was determined to be relatively fit based on the values of all other indices (Table 3).

The results on direct and indirect effects in the hypo- 
Table 2. Correlations between Variables and Verification of Construct Validity

\begin{tabular}{|c|c|c|c|c|c|c|c|c|}
\hline \multirow{2}{*}{ Variables } & \multirow{2}{*}{$\begin{array}{c}\mathrm{OF} \\
\mathrm{r}(p) \\
\end{array}$} & \multirow{2}{*}{$\begin{array}{l}\mathrm{NC} \\
\mathrm{r}(p) \\
\end{array}$} & \multirow{2}{*}{$\begin{array}{c}\mathrm{IL} \\
\mathrm{r}(p) \\
\end{array}$} & \multirow{2}{*}{$\begin{array}{c}\text { PSMA } \\
\mathrm{r}(p) \\
\end{array}$} & \multirow{2}{*}{ AVE } & \multirow{2}{*}{$\mathrm{CR}$} & \multirow{2}{*}{ Skewness } & \multirow{2}{*}{ Kurtosis } \\
\hline & & & & & & & & \\
\hline Organizational factors & 1 & & & & .73 & .93 & -0.15 & 1.37 \\
\hline Nursing competence & $.63(<.001)$ & 1 & & & .75 & .92 & 1.11 & 1.14 \\
\hline Informal learning & $.57(<.001)$ & $.56(<.001)$ & 1 & & .76 & .93 & -0.09 & -0.10 \\
\hline $\begin{array}{l}\text { Patient safety management } \\
\text { activities }\end{array}$ & $.50(<.001)$ & $.62(<.001)$ & $.54(<.001)$ & 1 & .65 & .92 & -0.07 & -0.25 \\
\hline
\end{tabular}

$\mathrm{AVE}=$ average variance extracted; $\mathrm{CR}=$ construct reliability; $\mathrm{IL}=$ informal learning; $\mathrm{NC}=$ nursing competence; $\mathrm{OF}=$ organizational factors; PSMA=patient safety management activities.

thetical model are as follows (Table 3). Among a total of five paths, four paths were significant (Figure 2). The direct effects of organizational factors $(\beta=.42, p=.002)$ and nursing competency $(\beta=.34, p=.005)$ on informal learning were significant, with $48 \%$ explanatory power. The indirect effect of organizational factors on patient safety management activities was significant $(\beta=.11, p=.008)$, whereas its direct effect was non-significant $(\beta=.03, p=$ $.710)$. Both direct $(\beta=.49, p=.002)$ and indirect $(\beta=.09, p=$ .007 ) effects of nursing competency on patient safety management activities were significant. Moreover, the direct effect of informal learning $(\beta=.26, p=.013)$ on patient safety management activities was significant. The explanatory power of organizational factors, nursing competency, and informal learning with respect to patient safety management activities was $50 \%$.

\section{DISCUSSION}

In the present study, the mediating effect of informal learning on the relationships between the organizational factors, nursing competency, and patient safety management activities in novice and advanced beginner nurses were evaluated. The study results showed that the explanatory power of these factors on patient safety management activities was $50 \%$.

Informal learning partially mediated the relationship between nursing competency and patient safety management activities. Further, a strong direct effect of nursing competency on patient safety management activities was observed. Previous studies have revealed a strong correlation between nurse competency and patient safety management activities [15], and informal learning was influenced by personal and organizational dimensions [21]. Moreover, informal learning influenced task involvement (including organizational socialization) among novice and advanced beginner nurses [18]. Therefore, the results of the present study are consistent with those of previous studies. Nurses are in close contact with patients in the final stage of healthcare delivery processes and are responsible for identifying and managing patient safety-related concerns [26]. Therefore, understanding the competence level of nurses and providing them with training in vulnerable areas are crucial. The evaluation of nursing competency and patient safety management activities, which reflect the characteristics of the department, should be conducted annually, and should include not only knowledge and skills, but also include aspects on professionalism, management and leadership, relationships, and cooperation [16]. In order to improve knowledge, informal learning that combines problem-based learning through actual cases of patient safety accidents and simulation education to improve skills and leadership can be used. This is not formal learning with a set scenario, but given only the problem situation, the preceptor's mentoring, coaching, and instant discussion can be utilized according to the process of leading the problem solving by novice and advanced beginner nurses. In addition, reflection learning or interviews with department heads can be applied to improve professionalism as a nurse, and team-based learning can be included to promote relationships or cooperation [27]. As such, informal and unplanned informal education can improve learning through clinical experiences, especially critical thinking, through interaction with other members [20]. In particular, novice nurses work independently and generally take 8 to 12 months to adjust to the organization [28]; however, the average novice nurse training period is reported to be only 57 days [29]. Informal learning can further enhance the clinical experience and organizational socialization through critical thinking [18]. Therefore, if informal learning is used, it is expected that novice and advanced beginner nurses will be able to shorten the period of organizational socialization and work adaptation.

Informal learning has a complete mediating effect on 
Table 3. Verification of the Hypothetical Model

\begin{tabular}{|c|c|c|c|c|c|c|c|}
\hline $\begin{array}{l}\text { Endogenous } \\
\text { variables }\end{array}$ & $\begin{array}{c}\text { Exogenous } \\
\text { variables }\end{array}$ & $\mathrm{SE}$ & CR & $p$ & SMC & $\begin{array}{l}\text { Direct } \\
\beta(p)\end{array}$ & $\begin{array}{l}\text { Indirect } \\
\beta(p)\end{array}$ \\
\hline \multirow{2}{*}{$\begin{array}{l}\text { Informal } \\
\text { learning }\end{array}$} & Organizational factors & .42 & 4.50 & $<.001$ & \multirow[t]{2}{*}{.48} & $.42(.002)$ & \\
\hline & Nursing competence & .34 & 3.68 & $<.001$ & & $.34(.005)$ & \\
\hline \multirow{3}{*}{$\begin{array}{l}\text { Patient safety } \\
\text { management } \\
\text { activities }\end{array}$} & Organizational factors & .03 & 0.35 & .724 & \multirow[t]{3}{*}{.50} & $.03(.710)$ & \multirow{3}{*}{$\begin{array}{l}.11(.008) \\
.09(.007)\end{array}$} \\
\hline & Nursing competence & .49 & 4.88 & $<.001$ & & $.49(.002)$ & \\
\hline & Informal learning & .26 & 2.81 & .005 & & $.26(.013)$ & \\
\hline \multicolumn{2}{|c|}{ Goodness-of-fit statistics } & \multicolumn{6}{|c|}{$x^{2} / \mathrm{DF}(p)=2.17(<.001), \mathrm{AGFI}=.84, \mathrm{NFI}=.89, \mathrm{TLI}=.93, \mathrm{CFI}=.94, \mathrm{SRMR}=.04$} \\
\hline
\end{tabular}

AGFI=adjusted goodness of fit index; $\mathrm{CFI}=$ comparative fit index; $\mathrm{CR}=$ critical ratio; $\mathrm{DF}=$ degrees of freedom; NFI=normed fit index; $\mathrm{SE}=$ standard error; $\mathrm{SMC}=$ squared multiple correlation; $\mathrm{SRMR}=$ standardized root mean squared residual; $\mathrm{TLI}=$ Tucker-Lewis index.

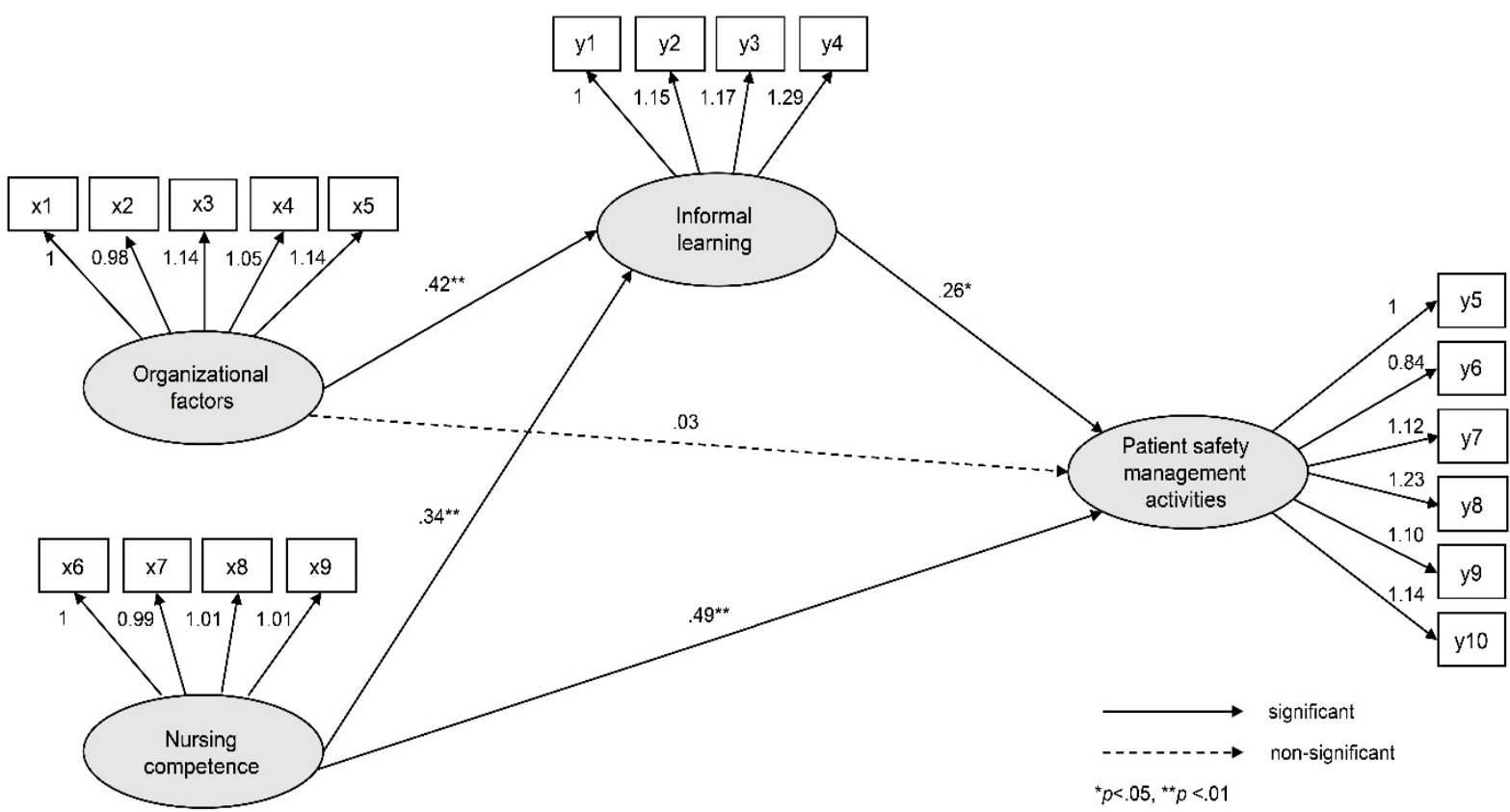

$x 1=$ safety management; $x 2=$ manager values; $x 3=$ system \& regulation, $x 4=$ safety investment; $x 5=$ safety training; $x 6=$ knowledge and skill; $x 7=$ professionalism; $x 8=$ management and leadership; $x 9=$ relationship and cooperation; $y 1=$ job knowledge acquisition; y2=organizational adaptation; $y 3=$ relationship formation; $y 4=$ self-development; $y 5=$ patient identification; $y 6=$ effective communication; $y 7=$ high-risk medication management; $y 8=$ accurate surgery/procedure identification; $y 9=$ infection prevention; y10 $=$ fall prevention.

Figure 2. Path diagram of the model.

the relationship between the organizational factors and patient safety management activities. Previous studies have reported that organizational factors did not directly affect behavioral changes [8,9]; however, they influenced patient safety management activities by mediating various factors, such as personal awareness [9] and attitude [8]. It can be seen that the organizational factor itself does not directly affect behavioral change, and personal factors such as knowledge, perception, and attitude act as antecedent factors [11]. In particular, informal learning is influenced by environmental components, including the safety system and regulation of the organization, manager val- ues, and safety training. An organization must establish efficient systems and regulations regarding patient safety management, and managers must understand the importance of patient safety and be willing to improve patient safety. Informal learning is difficult in organizations with an authoritative and hierarchical culture [18]. Accordingly, to promote patient safety management activities, an organizational environment that invigorates informal learning among the members through various means, such as voluntary reporting, implementation of patient safety systems, posters on patient safety management activities, practical training on patient safety, and intradepartmental 
discussions on the types and handling of safety incidents, must be fostered.

Since nurses provide the medical services closest to patients, patient safety management activities should be considered a top priority in nursing work. This study is meaningful in that it provides the fundamental data of an educational program that could improve patient safety management activities for novice and advanced beginners who are unable to perform nursing tasks independently in clinical practice. In order to improve patient safety management activities for novice and beginner nurses, it is necessary to strengthen individual nursing competency and effectively utilize informal learning, while establishing an organizational safety system and management system.

\section{CONCLUSION}

This study identified factors affecting patient safety management activities among novice and advanced beginner nurses and proposed effective interventions to promote such activities. The study findings demonstrated that organizational factors (i.e., safety management, manager values, system and regulation, safety investment, and safety training) and nursing competency (i.e., knowledge and skill, professionalism, management and leadership, and relationship and cooperation) mediate informal learning to improve patient safety management activities among novice and advanced beginner nurses. The study has the following crucial implication. The findings can provide fundamental data on the need for establishing educational systems to improve patient safety management activities among novice and advanced beginner nurses, who cannot independently or easily perform nursing care in clinical settings.

However, the following limitations exist. In addition to organizational and nursing competencies, there may be other factors that could affect patient safety management activities (job demands, job stress etc.). In particular, additional factors such as competency related to patient safety should be considered in future studies. To overcome the limitation of the self-reported data, future research should include objective indices. For example, observational methods can be used to collect data, and experimental studies can be used to identify the effects of improving patient safety management activities over the period of informal learning. Based on the study findings, a systematic intervention program can be developed to promote patient safety management activities among novice and advanced beginner nurses.

\section{REFERENCES}

1. World Health Organization. Patient safety: Making health care safer. World Health Organization, 2017. [Internet]. [cited 2020. April 20] Available from: https://apps.who.int/iris/handle/10665/255507

2. Bagnasco A, Galaverna L, Aleo G, Grugnetti AM, Rosa F, Sasso L. Mathematical calculation skills required for drug administration in undergraduate nursing students to ensure patient safety: A descriptive study: Drug calculation skills in nursing students. Nurse Education in Practice. 2016;16(1):33-39. https://doi.org/10.1016/j.nepr.2015.06.006

3. Choi EY, Lee HJ, Ock MS, Lee SI. Development of Korean root cause analysis software for analyzing patient safety incidents. Quality Improvement in Health Care. 2018;24(1):9-22. https://doi.org/10.14371/qih.2018.24.1.9

4. Hwang JI, Chin HJ, Chang YS. Characteristics associated with the occurrence of adverse events: A retrospective medical record review using the global trigger tool in a fully digitalized tertiary teaching hospital in Korea. Journal of Evaluation in Clinical Practice. 2014;20(1):27-35.

https://doi.org/10.1111/jep.12075

5. Lin LC. Comparison of risk management in Taiwan and the USA. Journal of Nursing Management. 2006;14(3):222-226. https://doi.org/10.1111/j.1365-2934.2006.00559.x

6. Kang JH, Kang HY, Kwon SH, Kim BR, Kim IS. Patient safety and nursing. Paju, South Korea: Uihakseowon; 2010.

7. Kim HI. Effect of organizational factors on safety accidents: inquiry into new model [dissertation]. Seoul: Chung- Ang University; 2004.

8. Kim KJ, Han JS, Seo MS, Jang BH, Park MM, Ham HM, et al. Relationship between intra-organizational communication satisfaction and safety attitude of nurses. Journal of Korean Academy of Nursing Administration. 2012;18(2):213-221. https://doi.org/10.11111/jkana.2012.18.2.213

9. Scott LD, Rogers AE, Hwang W-T, Zhang Y. Effects of critical care nurses' work hours on vigilance and patients' safety. American Journal of Critical Care. 2006;15(1):30-37. https://doi.org/10.4037/ajcc2006.15.1.30

10. El-Jardali F, Dimassi H, Jamal D, Jaafar M, Hemadeh N. Predictors and outcomes of patient safety culture in hospitals. BMC Health Services Research. 2011;11(45):1-12. https://doi.org/10.1186/1472-6963-11-45

11. Kim, NY, Jeong, SY. Patient safety management activities of clinical nurse: A modified theory of planned behavior. Journal of Korean Academy of Nursing Administration. 2019;25(5): 384-392. https://doi.org/10.11111/jkana.2019.25.5.384

12. Sherwood G, Zomorodi M. A new mindset for quality and safety: The QSEN competencies redefine nurses' roles in practice. Nephrology Nursing Journal. 2014;41(1):15-22. 
13. Ha NS, Choi J. An analysis of nursing competency affecting on job satisfaction and nursing performance among clinical nurses. Journal of Korean Academy of Nursing Administration. 2010; 16(3):286-294. https://doi.org/10.11111/jkana.2010.16.3.286

14. Jang KS. A study on establishment of clinical career development model of nurses [dissertation]. Seoul: Yonsei University; 2000.

15. Kim MJ, Kim JK. A study on the relationships among perception about patient safety culture, patient safety competence, and safety nursing activities of emergency room nurses. The Journal of the Korea Contents Association. 2017;17(10):268-279. https:// doi.org/10.5392.JKCA.2017.17.10.268

16. Park MR, Kim NC. Development of a nursing competence measurement scale according to nurse's clinical ladder in general wards. Journal of Korean Academy of Nursing Administration. 2014;20(3):257-271.

https://doi.org/10.11111/jkana.2014.20.3.257

17. Cho J, Yoon DY, Han SH. The effects of informal learning on organizational commitment: The mediating effects of self-efficacy. Korean Journal of Business Administration. 2018;31(8): 1527-1547. https://doi.org/10.18032/kaaba.2018.31.8.1527

18. Kim NY, Woo CH. Mediating effect of self-efficacy in the relationship between informal learning, shared leadership and organizational socialization of beginner and advanced beginner nurses. Journal of Korean Academy of Nursing Administration. 2018;24(1):1-9. https://doi.org/10.11111/jkana.2018.24.1.1

19. MoonSY, Na SI. Variables associated with informal learning of workers in small and medium-sized enterprises. Andragogy Today. 2011;14(3):111-138.

20. Marsick VJ, Watkins K. Informal and incidental learning in the workplace (Routledge Revivals). London: Routledge; 2015. https://doi.org/10.4324/9781315715926
21. Park HS, Lee C. Integrative literature review on factors influencing informal learning. Journal of Lifelong Education. 2012; 18:213-246.

22. Gong HH, Son Y-J. Impact of nurses' job satisfaction and organizational commitment on patient safety management activities in tertiary hospitals. Journal of Korean Academy of Fundamental Nursing. 2012;19(4):453-462. https://doi.org/10.7739/jkafn.2012.19.4.453

23. Carayon P, Hundt AS, Karsh B, Gurses AP, Alvarado, CJ, Smith M, et al. Work system design for patient safety: The SEIPS Model. BMC Quality \& Safety. 2006;15(suppl 1):i50-i58. https://doi.org/10.1136/qshc.2005.015842

24. Mitchell RJ. Path analysis: pollination. In: Scheiner SM, Gurevitch J, editors. Design and Analysis of Ecological Experiments. 2nd ed. New York: Oxford University Press; 2001. p. 217-234.

25. Park HH. A structural model of nurses' patient safety management activities [dissertation]. Daejeon: Eulji University; 2013.

26. Glancy GD, Chaimowitz G. The clinical use of risk assessment. Canadian Journal of Psychiatry. 2005;50(1):12-17. https://doi.org/10.1177/070674370505000104

27. Jang KS, Kim HY, Kim NY. A study for development of competency-based continuing education system depending on nurses' clinical ladder. The Journal of the Korea Contents Association. 2018;18(12):503-515.

https://doi.org/10.5392/JKCA.2018.18.12.503

28. Shin YW, Lee H, Lim Y. Predictors of clinical competence in new graduate nurses. Journal of Korean Academy of Nursing Administration. 2010;16(1):37-47. https://doi.org/10.11111/jkana.2010.16.1.37

29. Sin S, Park YW, Kim M, Kim J, Lee I. Survey on the education system for new graduate nurses in hospitals: Focusing on the preceptorship. Korean Medical Education Review. 2019;21(2): 112-122. https://doi.org/10.17496/kmer.2019.21.2.112 